\title{
Quiste óseo aneurismático de falange media de dedo anular con recidiva a dos años
}

\author{
Aneurysmal bone cyst in the middle phalanx of the ring finger 2 year recurrence \\ Ubaldo Ayala Gamboa, ${ }^{\star}$ Alejandro Miravete Gálvez, ${ }^{\ddagger}$ Alejandro Guillermo Gallegos Tejeda, ${ }^{\ddagger}$ Janet Pineda Díaz§
}

\section{RESUMEN}

El quiste óseo aneurismático es un tumor benigno poco frecuente que representa $9 \%$ de los tumores óseos. Sólo de 3 a $5 \%$ involucran la mano; de éstos, $52 \%$ aparecen en los metacarpianos y sólo $36 \%$ en las falanges. Presentamos el caso de una mujer de 52 años, cuyo padecimiento inició seis meses previos a su ingreso al recibir contusión directa en el dedo anular de la mano izquierda contra una pared, presentó dolor insidioso 2/10 en la EVA que se mantuvo constante. A la exploración física se encontró hiperflexión y dolor ligero a la extensión de la articulación interfalángica proximal y distal del anular, sin compromiso neurovascular y con arcos de movilidad de los dedos completos. Se realizó estudio radiográfico con placas simples en los que se encontró imagen radiolúcida en canal medular de la falange media del dedo anular, con características expansivas y evidente adelgazamiento de las corticales. Se decidió realizar tratamiento quirúrgico con resección de la lesión, crioterapia y aplicación de injerto óseo. El tejido recolectado fue enviado al Servicio de Patología Quirúrgica; el diagnóstico definitivo fue un quiste óseo aneurismático. Dos años después, la paciente tiene recurrencia del tumor en el mismo sitio. Se decide resección de la falange media en bloque y reconstrucción con injerto autólogo de cresta iliaca de falange media y artrodesis de las articulaciones interfalángicas proximal y distal del dedo anular izquierdo. La baja frecuencia de esta patología hace de interés su reporte y actualización en herramientas diagnósticas y terapéuticas, ya que es importante estar familiarizado con los diferentes tumores óseos de la mano, pues su tratamiento es diferente y los diagnósticos erróneos aumentan las tasas de recurrencia y la necesidad de reintervenciones quirúrgicas.

Palabras clave: Quiste óseo aneurismático, tumor óseo benigno, falange, mano, lesión lítica.

Nivel de evidencia: IV

\begin{abstract}
Aneurismatic bone cysts are rare benign tumors that represent about $9 \%$ of bone tumors. Only 3 to $5 \%$ involve the hands, of these $52 \%$ involve metacarpal bones and $36 \%$ involve phalanxes. We present the case of a 52 years old woman who suffered six months prior to her hospitalization a hand contusion to the left ring finger, causing insidious pain of 2/10 in the VAS pain. On physical examination, we found proximal and distal interphalangeal joints hiperflexion of the left ring finger with mild pain at extension, full motion arcs of the uninvolved fingers, and no neurovascular affection. $X$ rays showed a radiolucid image in the medullar cavity of the middle phalanx of the left ring finger, with expansive borders and evident thinning of the cortical layers. We decided to perform a surgical resection of the lesion and sent the collected material to the surgical pathology department, which diagnosed an aneurismatic bone cyst. The cyst recurred two years after first procedure. We decided to perform whole phalanx resection and reconstruction with bone autograph with arthrodesis proximally and distally. It is important to report such cases and update its diagnostic tools and treatment because of its low incidence, and clinicians should be aware of this and other differential diagnosis that must be accurately identified, since its treatment differs importantly and a misdiagnose may cause recurrence of such lesions and multiple surgical interventions that undermine the patient's quality of life.
\end{abstract}

Keywords: Aneurismatic bone cyst, benign bone tumor, phalanx, hand, lytic lesion.

Level of evidence: IV
* Cirujano de Mano.

‡ Residente de Traumatología y Ortopedia.

$\S$ Residente de Anatomía Patológica.

Centro Médico ABC.

Recibido para publicación: 06/01/2020. Aceptado: 03/03/2020

\section{Correspondencia: Alejandro Miravete Gálvez \\ Luis Cabrera Núm. 85, Economistas, Col. Ciudad Satélite, 53100, \\ Naucalpan de Juárez, Estado de México. Tel: 5513394331 \\ E-mail: dr.miravere@gmail.com}

Abreviaturas:

QOA = Quiste óseo aneurismático.

$E V A=$ Escala visual análoga

$\mathrm{AP}=$ Anteroposterior. 


\section{INTRODUCCIÓN}

El quiste óseo aneurismático (QOA) es un tumor benigno poco frecuente que representa $9 \%$ de los tumores óseos. Es localmente agresivo y se caracteriza por generar lesiones líticas y expansivas en hueso metafisiario, tanto del esqueleto axial como apendicular. ${ }^{1-3}$ Es más común en las dos primeras décadas de la vida y en el sexo femenino. Involucra principalmente los huesos largos de la extremidad inferior y los elementos de la columna vertebral y sólo de 3 a $5 \%$ involucran la mano; de éstos, $52 \%$ aparecen en los metacarpianos y $36 \%$ en las falanges., ${ }^{1,3-6}$

La patogénesis de estos tumores es incierta, aunque por mucho tiempo se atribuyó a la circulación venosa anormal que produce hemorragia intraósea y subperióstica, con activación de osteoclastos, reabsorción y remodelación ósea. En la actualidad, esta teoría sólo se acepta para los secundarios a otros tumores óseos, que representan hasta $30 \%$ de los QOA. ${ }^{1,5,7}$ Sin embargo, en los de tipo primario se ha encontrado un rearreglo del oncogén USP6 del cromosoma 17 , con la translocación t $(16 ; 17)$ (q22;p13), anormalidad ausente en los secundarios. ${ }^{8}$ La evolución natural del QOA implica crecimiento y destrucción local, pero no se considera una lesión premaligna. ${ }^{9}$

Debido a su baja incidencia, el médico suele desconocer el adecuado abordaje diagnóstico, tratamiento y pronóstico de estas lesiones. ${ }^{1,3,5}$

\section{CASO CLÍNICO}

Mujer de 52 años, sin antecedentes médicos de importancia. Inició su padecimiento aproximadamente seis meses previos a su ingreso al recibir contusión directa en dedo anular de mano izquierda contra una pared, con dolor insidioso 2/10 en la EVA que se mantuvo constante. En las últimas semanas refiere aumento de volumen y dolor progresivo en dedo afectado, por lo que decide acudir a consulta con médico especialista.

En la exploración física se encuentra aumento de volumen en región de falange media del dedo anular izquierdo, sin cambios de coloración o temperatura, hiperflexión y ligero dolor a la extensión de articulación interfalángica proximal y distal del anular izquierdo, con arcos de movilidad de los dedos completos y estado neurovascular distal conservado.

Fueron solicitadas radiografías AP, lateral y oblicua de la mano en las que se encontró una imagen radiolúcida del canal medular de la falange media del dedo anular izquierdo, con características expansivas y evidente adelgazamiento de las corticales (Figura 1).

Por los hallazgos clínicos y de imagen se decidió realizar tratamiento quirúrgico. Se accedió a la lesión con un abordaje dorsal, en la cual se encontró una cortical claramente adelgazada y de consistencia blanda. Se realizó corticotomía de la falange media, se halló cavidad con líquido francamente hemático, seguido de desbridamiento y curetaje con aplicación de crioterapia intracavitaria. Se envió el tejido recolectado al Servicio de Patología Quirúrgica para su diagnóstico definitivo (Figuras 2 y 3 ).

En el defecto secundario a la lesión se colocó injerto autólogo de hueso esponjoso de radio distal. Se inmovilizó a la paciente con una férula de yeso dorsal para dedo anular en extensión por cuatro semanas.

Histopatológicamente se encontró una lesión compuesta por cavidades llenas de elementos sanguíneos, sin revestimiento epitelial, limitadas por células fusiformes, múltiples células gigantes multinucleadas tipo osteoclasto y hueso trabecular reactivo con reacción osteoblástica, con un diagnóstico final de quiste óseo aneurismático (Figura 4).

Dos años después del primer evento quirúrgico y del seguimiento del caso, la paciente vuelve a presentar dolor de tipo urente e hiperalgesia en región de falange media de dedo anular izquierdo con intensidad EVA 6/10, así como crecimiento de la región de manera progresiva. El aumento de volumen fue limitando los movimientos y la empuñadura correcta de la mano. Debido al antecedente y la limitación en actividades de la vida diaria, decide acudir a valoración. Durante la exploración física se encuentra con aumento de volumen limitado a la región de falange media de anular izquierdo. La articulación interfalángica proximal tiene una flexión de $90^{\circ}$ y extensión completa sin deficiencia. La articulación interfalángica distal tiene una flexión de $20^{\circ}$ y extensión completa sin déficit. A la palpación se encuentra con aumento de volumen indurado.

Debido a estos hallazgos, se solicita realizar una resonancia magnética de mano. El reporte de patología menciona en dicho sitio «lesión lítica que involucra casi por completo la falange media del cuarto dedo, multiloculada, con niveles líquido-líquido por contenido hemático a considerar quiste óseo aneurismático, en una pequeña porción volar con discontinuidad-ruptura de la cortical»; además, se observan datos de extensión intraarticular.

Tras la valoración clínica, los antecedentes y el hallazgo de imagenología, se decide volver a inter- 
An Med (Mex) 2020; 65 (1): 51-56

venir quirúrgicamente. Se realiza abordaje dorsal de falange media de anular izquierdo. Se observa tejido adiposo en superficie cubital de color pardo, por lo que se disecan con cuidado los paquetes neurovasculares y se observa tumor que infiltra tendones extensores y de flexor profundo. Así también se observa infiltración en la articulación interfalángica proximal. Con dichos hallazgos se opta por una resección en bloque de falange media con quiste óseo

Figura 1:

Radiografía lateral de dedo anular de mano izquierda.
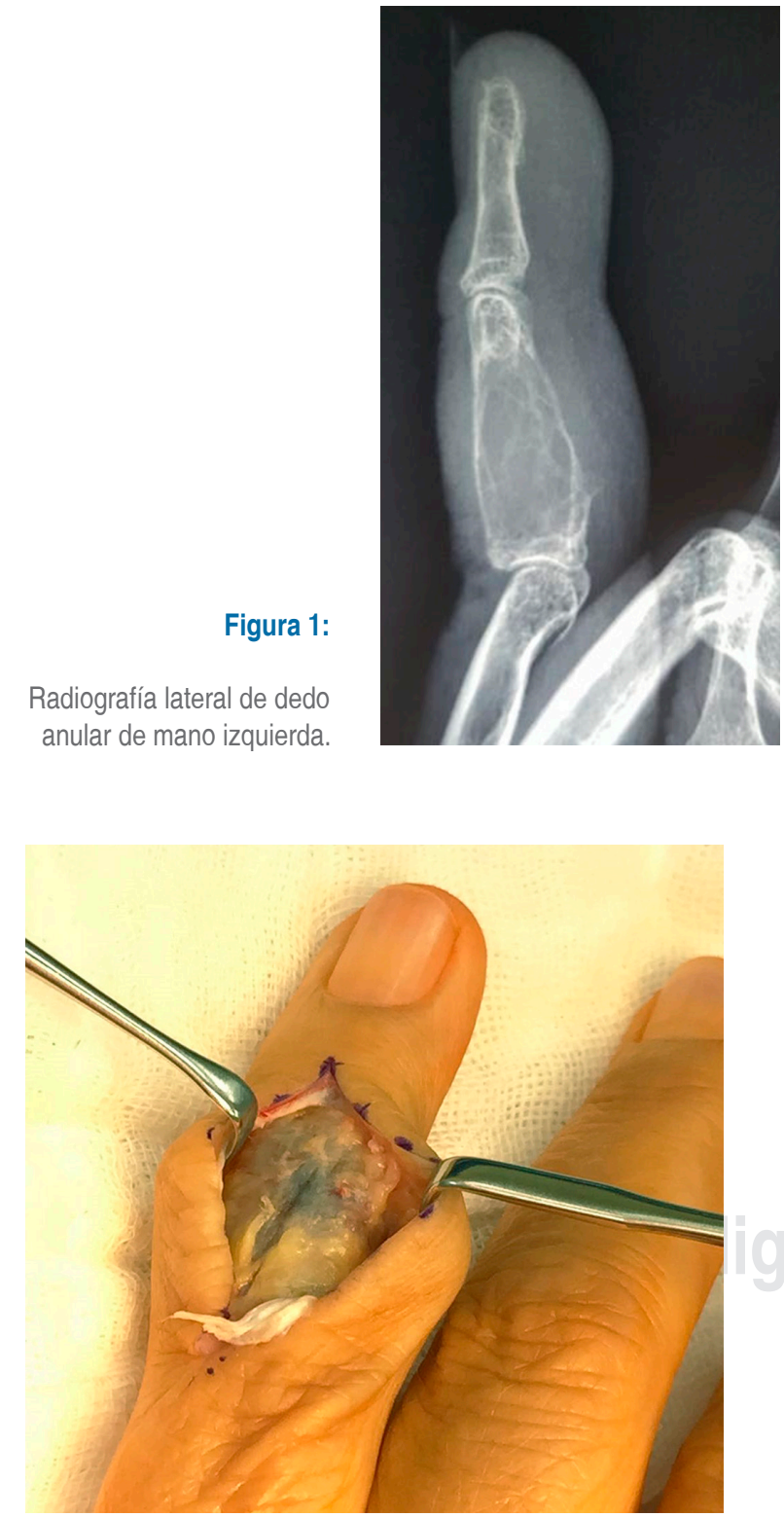

Figura 2: Exposición de lesión mediante un abordaje dorsal de falange media del dedo anular de mano izquierda, observando reblandecimiento importante de cortical dorsal de la falange media y coloración azulada.

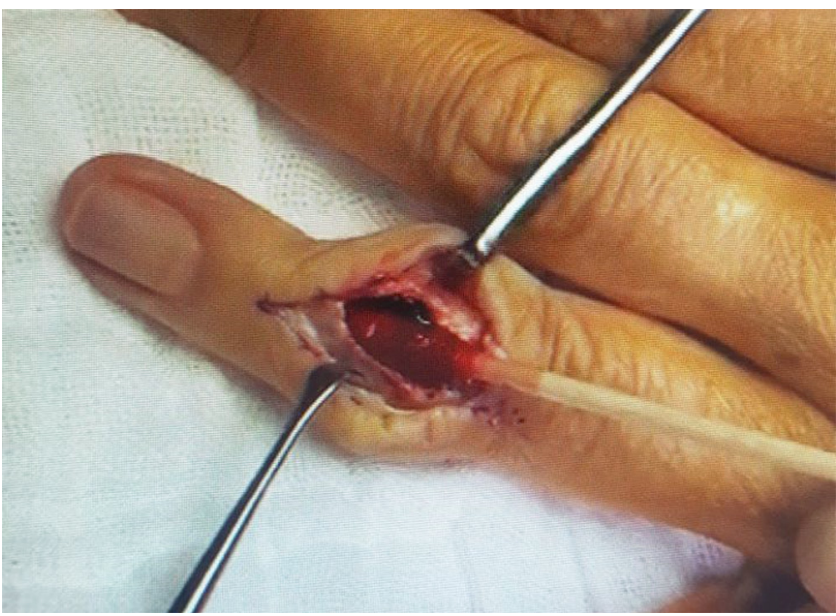

Figura 3: Se observa corticotomía dorsal de la falange media y cavidad con líquido francamente hemático.

aneurismático de anular izquierdo. Se realizó control de bordes sangrantes con radiofrecuencia para evitar diseminación del tumor. Después, se toma el injerto tricortical autólogo de cresta iliaca izquierda. Con bordes libres de tumor se coloca injerto de $4 \times 1 \mathrm{~cm}$ para reconstrucción de falange media y artrodesis de articulación interfalángica proximal a $40^{\circ}$ de flexión con placa en «T» de 2.3 x 1.3 de 11 orificios. Se coloca férula antebraquiopalmar en posición funcional con ferulización de dedo medio y anular izquierdo (Figuras $5 A$ y B).

\section{DISCUSIÓN}

El QOA fue descrito por primera vez en 1942 por Jaffe-Lichtenstein. ${ }^{10}$ Es una lesión poco común, muy rara en la extremidad superior y representa menos de 5\% en la mano. ${ }^{4}$ Aunque puede afectar a personas de cualquier edad, es más común en las primeras dos décadas de la vida y ligeramente mayor en el sexo femenino. ${ }^{4}$ Según nuestro conocimiento, existen en la literatura sólo tres series de casos de QOA en la mano, todos ellos con una muestra pequeña. También hay varios reportes de caso, uno de ellos es de nuestro centro médico. ${ }^{3,4}$

El primer estudio de gabinete que debe solicitarse y que brinda la primera clave para el diagnóstico del QOA es la radiografía simple. Clásicamente se encuentra una lesión quística radiolúcida, circunscrita, en una capa delgada de hueso cortical, comúnmente con trabéculas intralesionales que dan una imagen coloquialmente conocida como «burbuja de jabón». ${ }^{11}$ 
An Med (Mex) 2020; 65 (1): 51-56

La resonancia magnética puede evidenciar niveles de líquido que en los QOA representan las capas de sangre con distinta densidad, una sobre otra; también revela los septos intralesionales y edema perilesional. ${ }^{11}$

No obstante, es importante que el médico considere que estas características en los estudios de imagen no son exclusivas del QOA, sino que están presentes en otras entidades como el tumor de células gigantes y el osteosarcoma telangiectásico. ${ }^{7}$ Por esta razón, el diagnóstico definitivo se realiza sólo con el estudio histopatológico de una biopsia, que puede tomarse con trócar o, preferentemente, de la resección completa de la lesión. ${ }^{12}$

Macroscópicamente, el QOA consiste en múltiples cavidades anastomóticas de diámetro variable, desde milímetros hasta $2 \mathrm{~cm}$, que contienen sangre no coagulada y líquido seroso hemático. Las cavidades están delimitadas por septos fibrosos que se pueden calcificar en lesiones antiguas. El hueso cortical es delgado o ausente y se reemplaza por tejido de osteogénesis. Microscópicamente se observan cavernas sin recubrimiento epitelial llenas de sangre, delimitadas por septos fibrosos, fibroblastos e inflamación linfohistiocítica, con células gigantes tipo osteoclasto, osteogénesis reactiva y algunas mitosis. ${ }^{11,12}$

Desde las primeras descripciones del QOA realizadas por Jaffe-Lichtenstein, el tratamiento ha consistido en el curetaje y aplicación de injerto óseo. ${ }^{10}$ Sin embargo, la literatura reporta una tasa de recurrencia de hasta $60 \%$ cuando se utiliza el desbridamiento y curetaje como monoterapia. . $^{1,3,5,8,9,11}$
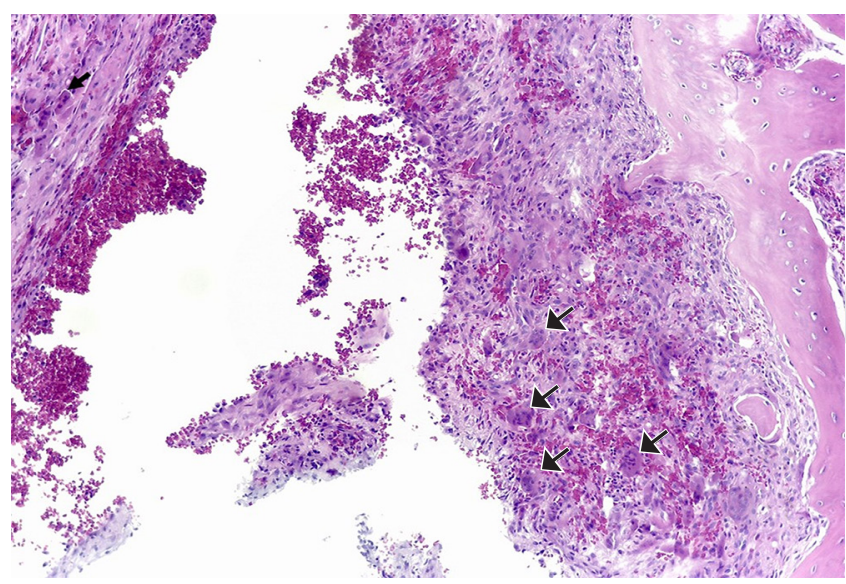

Figura 4: Se observa una cavidad con elementos sanguíneos sin revestimiento epitelial, rodeada por células fusiformes y células gigantes multinucleadas tipo osteoclasto (flechas negras).

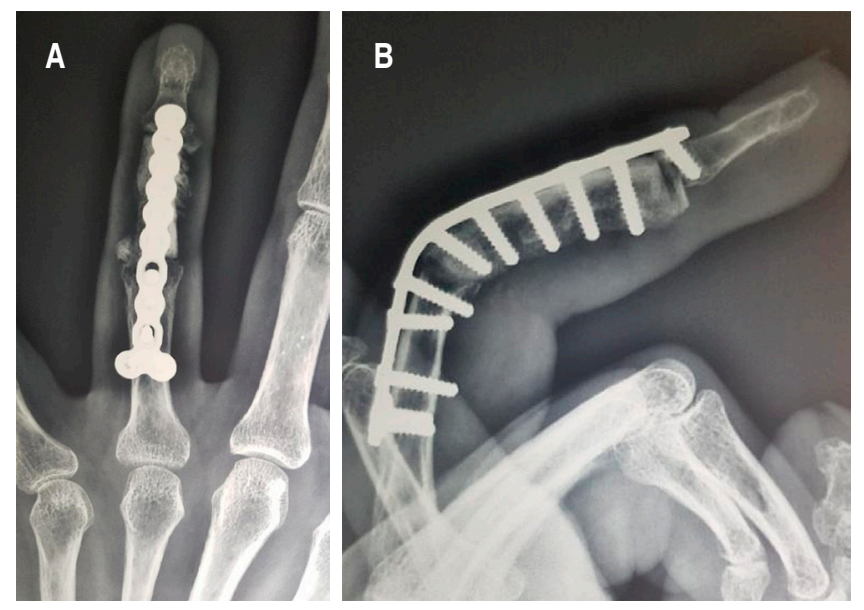

Figura 5: Radiografías postoperatorias de la reconstrucción con injerto autólogo de cresta iliaca, placa y tornillos.

En la actualidad se recomienda el uso de terapia adyuvante, incluyendo el fresado de alta velocidad, el metilmetacrilato, haz de argón, fenol o la crioterapia, que junto con el curetaje han reducido la recurrencia a 5-20\%, según la terapia adyuvante utilizada. ${ }^{1,7,8,11,12}$

Los datos clínicos y las imágenes radiográficas de éstas y otras lesiones suelen ser difíciles de diferenciar, en especial con el tumor más común de la mano, el encondroma. Consideramos de vital importancia que el cirujano de mano esté totalmente familiarizado con las diferentes tumoraciones de la mano y con su tratamiento, pues es común programar la escisión quirúrgica sin contar con el instrumental y material necesario para realizar un adecuado tratamiento en caso de enfrentarnos a un tumor diferente al encondroma. Los autores concordamos en que es importante tener una exposición completa de la lesión intracavitaria por medio de la apertura de una ventana amplia en el hueso, que permita el uso cómodo y adecuado del instrumental de curetaje y desbridamiento para lograr una resección total de la lesión. El uso de terapia adyuvante como la crioterapia debe usarse con reserva, ya que aunque Peeters y colaboradores reportan que ésta reduce la recurrencia de los QOA a sólo 5\% de los casos, el estudio es retrospectivo y con una muestra poco representativa. ${ }^{12} \mathrm{El}$ resto de la literatura acerca del uso de la crioterapia incluye sólo reportes de casos clínicos, sin existir hasta el momento un ensayo con muestra significativa o un metaanálisis que brinden evidencia contundente acerca de los beneficios del uso de este tipo de terapias para 
An Med (Mex) 2020; 65 (1): 51-56

el QOA, por lo que consideramos necesario realizar más estudios al respecto para conocer con mayor certeza la incidencia y tasa de recurrencia de este tipo de lesiones.

La información en cuanto a recidiva del QOA es controvertida. Crowe y colaboradores reportan una tasa de recurrencia baja con curetaje de la lesión. Sin embargo, ellos mismos lo reportan como un hallazgo contrario a lo visto en otras series en las que la recidiva es de hasta $20 \%$ de los casos de QOA en otros huesos del esqueleto. Crowe de misma forma, refiere que la recidiva se ve poco después de la cirugía primaria. Mankin reporta que el QOA tratado mediante curetaje y chips óseos recidiva hasta un $21 \%$, mientras que la resección o escisión de la lesión agregada con injerto autólogo tiene una recidiva de $11 \%$. Sin embargo, este último artículo incluye QOA en otros huesos del esqueleto y no incluye pacientes con huesos de la mano involucrados. ${ }^{3,13}$

El tratamiento de la recurrencia se basa en curetaje repetitivo con terapia adyuvante o en la resección de la lesión y reconstrucción. Debido a la baja incidencia de casos reportados de recurrencia en mano y de su tratamiento, aún no se puede determinar el tratamiento definitivo que se debe recomendar hacer. Según Mankin, el procedimiento que no reportó recurrencia del QOA fue la resección o escisión de la lesión con injerto análogo, fue reportado un $0 \%$ de recurrencia. ${ }^{3,13,14}$

Debido a la recurrencia reportada, los tratamientos se van volviendo más agresivos, lo que implica una reconstrucción con mayor nivel de dificultad. Zaidenberg y colaboradores realizaron una resección en bloque de QOA en cuarto metacarpiano y realizaron reconstrucción con aloinjerto metacarpiano y uso de placa de bloqueo y compresión. Ocho años después en citas de seguimiento, el paciente había recuperado un $85 \%$ de la fuerza de empuñadura, comparada con mano contralateral. El dolor en reposo en EVA fue reportado 0/10 con retorno al trabajo y a realizar actividades de la vida diaria sin limitaciones ni dolor. Las radiografías de seguimiento no demuestran recurrencia del QOA. ${ }^{15}$

Según Nanda y colaboradores, no pueden concluir el mejor manejo para el QOA en mano, debido a la baja incidencia que hay. Ellos concluyen que un tratamiento inicial insuficiente lleva a una recurrencia del tumor de mayor tamaño y que logra alcanzar estructuras articulares. Por ello, determinan que el tratamiento de elección debe ser resección en bloque. ${ }^{16}$

\section{CONCLUSIÓN}

Presentamos el caso de un QOA en una mujer de 52 años en la falange media del dedo anular izquierdo tras haber sufrido una contusión directa unos meses antes. Debido a que los datos clínicos de esta lesión son inespecíficos, resulta de vital importancia la realización de estudios de imagen que pueden sugerir su diagnóstico y nos auxilian en la planeación de la escisión quirúrgica. Sin embargo, el diagnóstico definitivo sólo puede realizarse por medio del estudio histopatológico del material obtenido del procedimiento, por lo que su envío al Servicio de Patología Quirúrgica resulta trascendental. Tener el correcto diagnóstico del QOA permitirá saber si la escisión quirúrgica fue curativa, así como conocer la probabilidad de recurrencia y reintervención, lo cual resulta relevante sobre todo en pacientes con comorbilidades que pueden tener complicaciones al ser sometidos a procedimientos invasivos. El uso de terapias adyuvantes, incluyendo la crioterapia, no se recomienda en todos los casos, pues no se cuenta por el momento con suficiente evidencia acerca de la disminución de recurrencias de este tipo de lesiones óseas.

\section{BIBLIOGRAFÍA}

1. Mavrogenis AF, Panagopoulos GN, Angelini A, Lesenský J, Vottis C, Megaloikonomos PD et al. Tumors of the hand. Eur J Orthop Surg Traumatol. 2017; 27 (6): 747-762.

2. Tarazona-Velutini P, Romo-Rodríguez R, Saleme-Cruz J. Quiste óseo aneurismático en falange proximal de dedo de mano. Caso clínico y revisión de la bibliografía. Acta Ortop Mex. 2012; 26 (4): 245-249.

3. Crowe MM, Houdek MT, Moran SL, Kakar S. Aneurysmal bone cysts of the hand, wrist, and forearm. J Hand Surg Am. 2015; 40 (10): 2052-2057.

4. Hakim DN, Pelly T, Kulendran M, Caris JA. Benign tumours of the bone: a review. J Bone Oncol. 2015; 4 (2): 37-41.

5. Havulinna J, Parkkinen J, Laitinen M. Aneurysmal bone cyst of the index sesamoid. J Hand Surg Am. 2005; 30 (5): 10911093.

6. Cottalorda J, Kohler R, Sales de Gauzy J, Chotel F, Mazda $\mathrm{K}$, Lefort $\mathrm{G}$ et al. Epidemiology of aneurysmal bone cyst in children: a multicenter study and literature review. J Pediatr Orthop B. 2004; 13 (6): 389-394.

7. Park H, Yang S, Sheppard W, Hegde V, Zoller S, Nelson S et al. Current management of aneurysmal bone cysts. Curr Rev Musculoskelet Med. 2016; 9 (4): 435-444.

8. Oliveira AM, Chou MM. USP6-induced neoplasms: the biologic spectrum of aneurysmal bone cyst and nodular fasciitis. Hum Pathol. 2014; 45 (1): 1-11.

9. Hsu CS, Hentz VR, Yao J. Tumours of the hand. Lancet Oncol. 2007; 8 (2): 157-166.

10. Jaffe HL, Lichtenstein L. Solitary unicameral bone cyst: with emphasis on the roentgen picture, the pathologic appearance and the pathogenesis. Arch Surg. 1942; 44 (6): 1004-1025. 
An Med (Mex) 2020; 65 (1): 51-56

11. Mascard E, Gomez-Brouchet A, Lambot K. Bone cysts: unicameral and aneurysmal bone cyst. E Orthop Traumatol Surg Res. 2015; 101 (1 Suppl): S119-S127.

12. Peeters SP, Van der Geest IC, de Rooy JW, Veth RP, Schreuder HW. Aneurysmal bone cyst: the role of cryosurgery as local adjuvant treatment. J Surg Oncol. 2009; 100 (8): 719-724.

13. Mankin HJ, Hornicek FJ, Ortiz-Cruz E, Villafuerte J, Gebhardt MC. Aneurysmal bone cyst: a review of 150 patients. J Clin Oncol. 2005; 23 (27): 6756-6762.
14. Başarir K, Saglik Y, Yildiz Y, Tezen E. Aneurysmal bone cyst of the hand: a report of four cases. Hand Surg. 2006; 11 (1-2): 35-41.

15. Zaidenberg EE, Farias Cisneros E, Miller R, Zaidenberg CR. Metacarpal aneurysmal bone cyst en bloc resection reconstructed with osteoarticular allograft. J Hand Surg Am. 2019; 44 (5): 425.e1-425.e5.

16. Nanda SN, Tripathi S, Shiraz SM, Warrier S. Aneurysmal bone cyst of 3rd metacarpal, management and follow-up: a case report. J Orthop Case Rep. 2018; 8 (2): 9-12. 